\title{
Research on the Interactive Design of Educational Apps Oriented to Preschoolers from the Perspective of Flow Experience

\author{
Jing-Hua Han ${ }^{1}$ and Qi Cao* \\ 1hanjing013@126.com, 329605718@qq.com \\ *The Corresponding author
} \\ ${ }^{1}$ Department of Digital Art, Beijing Forestry University, Beijing, China
}

Keywords: Flow experience; Preschoolers; Educational Apps; Interactive design

\begin{abstract}
Focused on the design of Apps oriented to preschoolers, this paper analyzed the preconditions to get users into flow from the perspective of flow experience, and explored interactive designs arousing flow experience by means of investigating relationships among clear and explicit learning goals, immediate and effective feedback and balance between learning difficulties and capabilities, in an attempt to enhance educational Apps oriented to preschoolers.
\end{abstract}

\section{Introduction}

In the 1970s, Mihaly Csikszentmihalyi, an American psychologist, discovered that when engaged in what really interests them people tend to experience a unique feeling making them immersed in the job in hand while forgetting to sleep and eat. They usually take great pleasure in doing it and often show amazing innovations [1]. Such experience was called as Flow. These days with the rapid development of mobile intelligent terminals, greater and greater importance are attached to user experience while designing internet products, into which Flow, as a measure of user experience, has been embedded.

Preschool education has always been a topic of concern among parents. In contemporary society, with the booming of Apps for children education, mobile devices like iPads are popular with young parents when carrying out early childhood education at home. In 2013, Apps in App Store amounted to 1237119 in total, among which 95065 were related to education. Moreover, $80 \%$ of the educational Apps were for children [2]. However, educational Apps are not always beneficial. Inferior ones do exist. The combination of Flow Theory and product designs at the very beginning when developing the Apps will refine the specification and classification of elements concerning flow. The application to designs is likely to generate controllable models or mechanisms helping children to get into flow and thus make learning itself interesting for children.

According to Csikszentmihalyi Theory, prerequisites for flow are as follows: 1) clear and explicit goals; 2) immediate and effective feedback; 3) balance between challenges and skills. This paper took into account of the preconditions above and combined the interactive designs of educational Apps to explore the application of Flow Theory to educational Apps aimed at children.

\section{Clear and Explicit Learning Goals}

Csikszentmihalyi holds the view that when users are fully immersed in the activities, their irrelevant perceptions and thoughts will be filtered out for they are likely to be involved into a shared empirical mode characterized by narrow focus and loss of self-consciousness in which they only respond to explicit goals and concrete feedbacks[3]. Therefore, explicit goals play a vital role in helping users to experience flow.

Explicit leaning tasks. Preschoolers refer to infants aged between three and six years old, who have yet not developed fully who are in a special physiological and mental stage. Moreover, their logical thinking styles are usually weak, incompetent to analyze or tackle sophisticated problems. Educational Apps aimed at these children are supposed to be explicit in training contents based on the preschool education system and specific in functions through detailed classification of the product types at the very beginning of designing. Combinations of explicit learning tasks and 
corresponding situations make learning goals explicit. For example, tasks involving assisting virtual roles in brushing away the moth will instruct children to brush and foster a good habit of brushing teeth.

Simple interactive operations. Preschoolers are weak in information processing and often find it difficult to search, classify and apply information. Concise interactive interface, simple information structure can reduce interferences from irrelevant factors and decrease the interactive difficulty for children. What's more, sophisticated gestures are unreachable for children at this stage. Simple and repetitive gestures, like clicking, sliding and touching, will reduce the disappointment and resistance in operation and make them better master interactive approaches, conducive to flow.

Instructive interactive approaches. Appropriate use of instructive interactive-designs such as colors, voices and prompt signs will provide children with explicit interactive goals so as to help them find correct interactive approaches.

Preschoolers' eyes are still under development. The absorptivity of crystalline lens of eyeballs is less than $10 \%$. Such a physiological feature makes children at this stage prefer bright, pure and contrasting colors[4]. Therefore, interactive icons based on this assumption will be more attention-drawing for children so as to guide them to click and touch more. Voice guidance is like parents' companion to some extent. Informing children of next steps in each task and offering operational information help them successfully complete tasks to have a wonderful experience. Directive arrows, dynamic signs and twinkling borders are all interactively suggestive. Use of such guiding information will make children learn of the goals and improve their chances of success so as to enhance confidence in interaction.

\section{Immediate and Effective Feedback}

Feedback is a response or direction to happened operational behaviors. Immediate and effective feedback mechanism allows users to know more about the progress, and caters for their needs for responses to their devotion, circumventing anxiety and desperation caused by deviations from the goals which may impede the flow experience[5].

Feedback from different sensorial organs. Compared with traditional educational media, Apps oriented to children education based on mobile devices provide good design environment for flow experience by means of its advantages in information processing and immediate feedback. Designs of visual, auditory and tactile senses offer users immediate and efficient feedback. Visual feedbacks help children perceive current learning and know the results. Auditory feedbacks arouse their learning interest and confidence through encouragement. With regard to touch sense, shaking feedbacks engage children's attention.

Reward mechanism. Rewards are a kind of feedback mechanism after evaluating users' operation. In psychology, rewards are regarded as a psychological process to stimulate motivation constantly With higher the stimulation level, more tasks will be accomplished. Preschoolers are poor in concentration, easily influenced by the environment due to frequent attention shift. The design of missions with virtual trophies or medals for winners can arouse children's enthusiasm, motivating them to make continuous attempts.

Quick Math Jr, as shown in Figure1, is an App to foster children's math intuition. One of the five sensory organs of the little monster will be awarded randomly for passing each task. The body of the little monster is a reward for the completion of a periodical learning. As long as having the body, children can make a new little monster in the manufacturing factory and have another learning partner. Unexpected rewards bring children more surprises and happiness so as to generate more active and positive emotions making learning interesting as well as providing them with internal driving forces. 


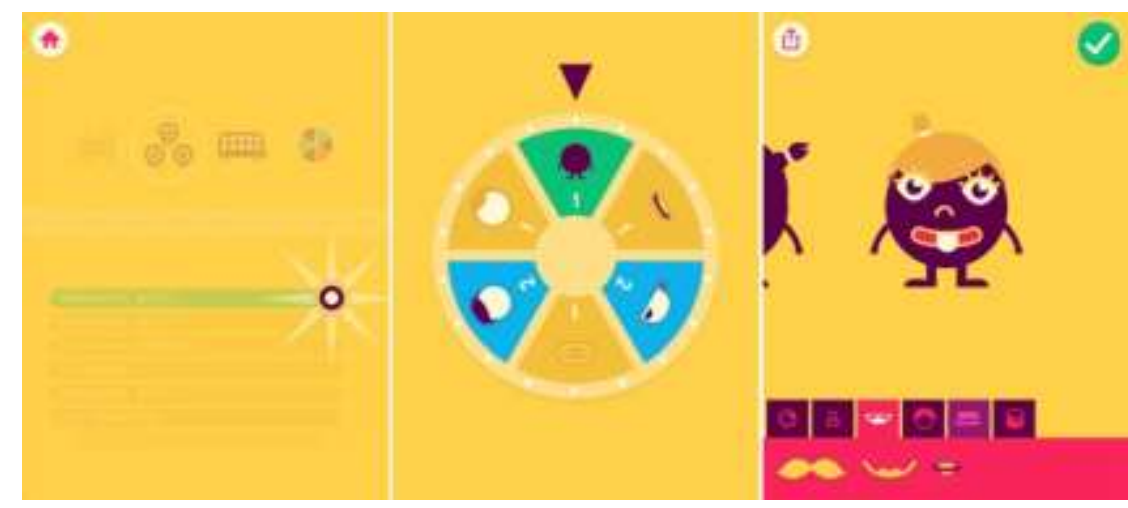

Figure1. 《Quick Math Jr》

\section{Balance Learning Difficulties and Learning Capabilities}

According to Flow Theory, challenges and skills are significant factors influencing users' immenseness in the experience. When the two factors reach a higher level of balance, users will feel the joy brought by the unification of will and activities [6]. Figure 2 demonstrates relationships between challenges and skills in the Flow Model, ideally explaining changing relations between skills and challenges with A, B, C and D representing different states of the users respectively. A indicates that users are less competent and likely to feel anxious since the challenges are difficult for them. C suggests that users are competent and tend to feel bored with challenges are so easy for them. In comparison, B and D imply that challenges and skills are kept in balance, located in the channel of Flow. However, more skills and challenges are needed for B. users at this point are experiencing real flow and more pleasure and excitement. In light of this, designs of Apps for educating preschoolers can bring children more flow experience through matching challenges with skills.

Flow areas fit for users. The core of educational Apps lies in instructing children. Hence educational products tailored for children are expected to be professional and scientific. It is essential to possess a professional team to design contents conforming to children's cognitive levels. For this, plenty of research should be conducted to make a thorough analysis of skills and abilities they are already equipped with, based on which difficulty levels for this group will be determined for appropriate areas of flow experience.

Progressive task difficulty. Through learning on Apps, children can achieve gradual and steady self-improvement. At the very beginning of learning, rapid increase in task difficulties will make children suffer setbacks and even lose confidence. Therefore, increase in difficulty should be steady to leave users more time so that they can learn how to operate and comprehend the rules, after which the difficulty will be enhanced with the increase in skills. In addition, transformations of large goals into small goals matching with current skill levels help children approach to the goals step by step during which they will feel the sense of achievement and be motivated to learn.

Leo's Pad, an educational App, divides telescopes into the following four parts in the stages of assembling props: light pipes, camera lens, focal tubes and ocular lens. Children have to piece each component together to complete the task. As shown in Figure 3, children need to piece a rectangle and two squares together. Then they need to piece two quadrants and one semicircle into a circular lens. To finish the third task they need to piece four triangles into a trapezoidal focal tube. in regard to the last task, they are required to piece two quadrants, two triangles and two trapezoids into an ocular lens. It is obvious that the take difficulty is ascending. Completion of the simple task brings fulfillment as well as leads children to discover rules of the game. The ascending difficulty in the following tasks not only poses challenges but also gets them in the flow through rising to different challenges. 


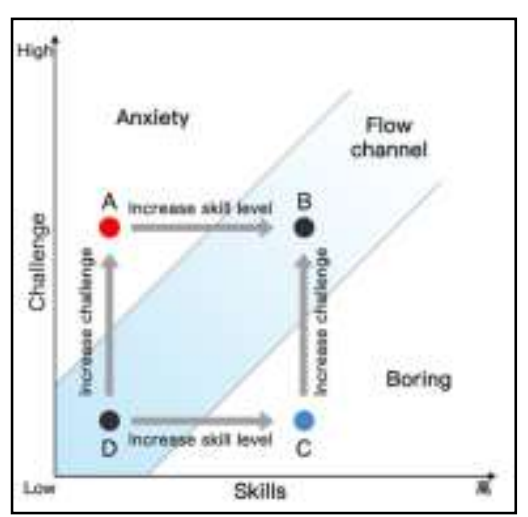

Figure2. Flow Theory

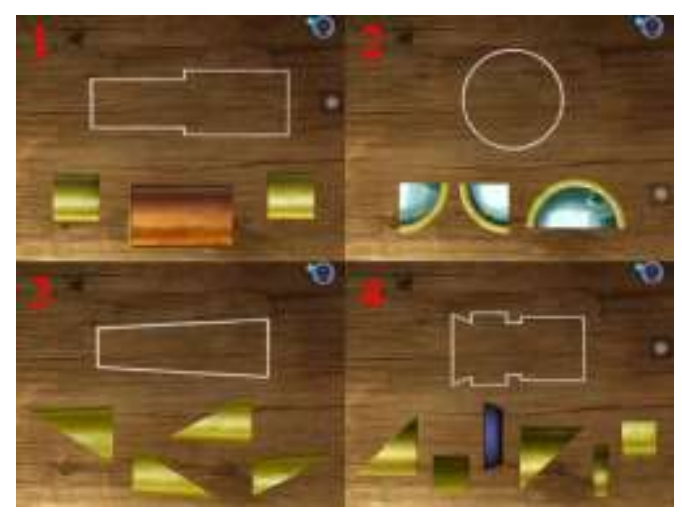

Figure3. 《Leo's Pad》

\section{Conclusion}

The digital age offers parents and children brand-new educational and learning models. The intervention of flow experience provides a new perspective. The integration of Flow Theory into designs of educational Apps oriented to preschoolers will make the products more attractive, promote user experience and provides children with maximum happiness and joy.

\section{Acknowledgements}

This work was supported by the Fundamental Research Funds for the Central Universities (2015ZCQ -YS-02) and Beijing Higher Education Young Elite Teacher Project (YETP0785).

\section{References}

[1] CSIKSZENTMIHALYI Mihaly, CHEN Xiu-juan Translate, Finding flow[M].Beijing:China CITIC Press,2009.

[2] http://learning.sohu.com/20140207/n394573068.shtml

[3] CSIKSZENTMIHALYI Mihaly. Play and Intrinsic Rewards[M]. Netherlands: Springer, 2014:15(3):135-153.

[4] LUO Hao-Tian. The Research on the Interactive Design about Children's Digital Picture Books [D]. Wuxi: Jiangnan University,2014.

[5] Mihaly Csikszentmihalyi. Play and Intrinsic Rewards[J]. Journal of Humanistic Psychology , 2014,15(3):41.

[6] HONG Liu, GUO Jia-yi, GE Shi-jun. Flow and User Experience Design[J]. Art and Design (Theory), 2009,(03):178-180. 